\title{
Impact of ABO-incompatibility on hepatic artery thrombosis in living donor liver transplantation
}

\author{
Seong Hoon Kim, Jangho Park, Sang Jae Park \\ National Cancer Center, Goyang-si, Gyeonggi-do, Republic of Korea \\ Contributions: (I) Conception and design: SH Kim; (II) Administrative support: SH Kim, SJ Park; (III) Provision of study materials or patients: All \\ authors; (IV) Collection and assembly of data: SH Kim, J Park; (V) Data analysis and interpretation: SH Kim, J Park; (VI) Manuscript writing: All \\ authors; (VII) Final approval of manuscript: All authors. \\ Correspondence to: Seong Hoon Kim, MD, PhD. Organ Transplantation Center, National Cancer Center, 323 Ilsan-ro, Ilsandong-gu, Goyang-si, \\ Gyeonggi-do 10408, Republic of Korea. Email: kshlj@hanmail.net; kshlj@ncc.re.kr.
}

Background: The current era of rituximab-based regimens brought improved survival in ABO-
incompatible (ABO-I) living donor liver transplantation (LDLT). Nevertheless, the actual risk for hepatic
artery thrombosis (HAT) still remains to be investigated. The aim of this study was to evaluate the impact of
ABO-incompatibility on HAT in adult LDLT patients. Methods: Patients who received ABO-I LDLT were compared to those who received ABO-compatible (ABO-C) LDLT with a special focus on HAT.

Results: A total of 378 patients underwent LDLT from January 2012 to February 2018. Of those, ABO-I LDLT was performed in 78 consecutive patients. The other 300 patients with ABO-C LDLT constituted the comparator group. No significant differences were observed between the two groups in baseline and perioperative characteristics. HAT occurred in 11 (2.9\%) patients, 2 and 9 patients in ABO-I and ABO-C LDLT groups, respectively, which didn't show any significant difference between the two groups $(\mathrm{P}=0.84)$. All were categorized into early HAT. Immediate revascularization by intraarterial thrombolysis was successfully employed in 8 patients. Surgical revision of anastomosis and retransplantation were performed in 1 and 2 patients, respectively. No one-year mortality was related to HAT in the two groups. The overall outcomes including biliary complications made no significant difference between the two groups.

Conclusions: ABO-incompatibility has no adverse impact on the incidence and treatment outcome of HAT in the current rituximab-based desensitization.

Keywords: Living donor liver transplantation (LDLT); hepatic artery thrombosis (HAT); ABO-incompatibility

Submitted Sep 17, 2019. Accepted for publication Oct 17, 2019.

doi: $10.21037 /$ atm.2019.11.34

View this article at: http://dx.doi.org/10.21037/atm.2019.11.34

\section{Introduction}

Hepatic artery thrombosis (HAT) is the most devastating vascular complication following liver transplantation that can result in graft loss with a high patient mortality of $33.3 \%(1)$.

In living donor liver transplantation (LDLT), reconstruction of hepatic arteries is a challenge because the graft hepatic artery is short in stump length, small in diameter, and sometimes discrepant in vessel size from the recipient hepatic artery, all of which may add to the risk of HAT. Aside from the causes of surgical technique, many nonsurgical risk factors have been implicated in the development of HAT, although the exact pathogenesis of this complication still remains unclear (2).

ABO-incompatible (ABO-I) LDLT can be a final expedient if no compatible donor is available. In the recent era of rituximab-based prophylaxis, acceptable outcomes has been reported in ABO-I LDLT (3-5) and, as of now, has become more prevalent with improved 
survival. However, the actual risk of HAT has not ever been investigated. There is real concern that the added desensitization needed for ABO-I LDLT may increase the risk of HAT following LDLT, because there was a report on acute venous thrombosis while on rituximab treatment (6). Moreover, rituximab caused myocardial infraction by inducing an extensive thrombus in the coronary artery. The proposed mechanism was the release of cytokines, which cause vasoconstriction, platelet activation, and/or rupture of atherosclerotic plaque (7). Plasmapheresis was also reported to be a potential danger of thrombosis (8). Furthermore, ABO-incompatibility was actually found to be an independent risk factor for early HAT (9). This possibly suggests another menacing nonsurgical cause.

To deal with this issue, this study was undertaken to elucidate the impact of ABO-incompatibility on the incidence and outcome of HAT by comparing between ABO-I and ABO-compatible (ABO-C) LDLTs.

\section{Methods}

\section{Patients and study design}

This is a retrospective review of medical records. The study comprised consecutive adult patients (18 years or older) who received ABO-I LDLT at National Cancer Center, Korea between January 2012 and February 2018.

In terms of the selection criteria both in donors and recipients, ABO-I LDLT were not different from ABO-C LDLT, which were previously described (10-13). The information on surgical risk and expected survival was given and understood by both all donors and recipients.

During the study period, a comparative analysis was conducted between patients with ABO-I LDLT and those with ABO-C LDLT. The study variables included basic patient characteristics, operative details, and underlying disease.

The duration of hepatic artery anastomosis was calculated from the time point of the first bite of suturing until hepatic artery reperfusion. Early HAT is defined as any thrombosis within 30 days of transplantation, whereas late HAT is defined as occurring 30 days later the transplantation $(14,15)$.

The Korean Network for Organ Sharing affiliated to the Ministry of Health and Welfare of Korea approved all LDLT procedures. The Institutional Review Board of National Cancer Center, Korea approved this study, and informed consent was waived because this was a retrospective study (IRB number: NCC2019-0087).

\section{Surgical procedure}

In all LDLTs, the right robe graft was utilized. A single attending surgeon (Seong Hoon Kim) was responsible for the orchestration and progress of all surgical procedures on donor and recipient. He performed hepatic artery anastomoses in all patients. The technical details of donor surgery were specified previously (16-21).

On recovering the liver graft, histidine-tryptophanketoglutarate solution was infused into the portal vein, and the hepatic artery was flushed by injection using a 24-gauge catheter.

After the native liver was removed in the recipient, hepatic vein anastomosis was performed. Venous branches of the middle hepatic vein and inferior right hepatic vein of the graft was reconstructed when they were larger than $5 \mathrm{~mm}$ in diameter. The graft portal vein was anastomosed to either right or main portal vein of the recipient according to its size and redundancy. After graft reperfusion, hepatic artery was anastomosed under microscopic guidance. Biliary reconstruction was performed with end-to-end duct-to-duct anastomosis.

\section{Operative technique of hepatic artery anastomosis}

The basic principle was as follows: direct end-to-end anastomosis (recipient hepatic artery to graft hepatic artery) with interrupted 8-0 or 9-0 polypropylene sutures using the double micro-clamp under an operating microscope with $10 \times$ magnification. The surgeon was positioned at the right side of the operating table and one assistant was at the left. One suction drain was placed at the right subphrenic space so that blood and ascites was continuously drained to clear the operating field. The branch of the recipient's hepatic artery was dissected as peripherally as possible. This dissection was then carried proximally to the bifurcation of the proper hepatic artery and on to the common hepatic artery. If necessary, the common hepatic artery was dissected and the gastroduodenal artery ligated and divided to facilitate mobilization. After selecting the appropriate size-matched vessel and clearing of the surrounding connective tissue of arterial external layer, the cutting edges of both arteries were arranged properly for anastomosis and fixed in a double clip. Angle sutures were placed at both edges and then tied. The other sutures between angle sutures were performed on the anterior wall of both 
recipient and graft arteries, and then were completed on the posterior wall in the same manner as in the anterior wall sutures by turning the double micro-clamp. The clamp was then removed to reestablish flow through the hepatic artery. The duration of hepatic artery anastomosis was calculated from the time point of the first bite of suturing until hepatic artery reperfusion. The total number of stitches ranged from 8 to 12 according to the arterial diameter. Sometimes, a decrease of the tidal volume or manual bagging instead of mechanical ventilation was used to secure stabilized operative field from large movement caused by respiration. The caliber discrepancy was dealt with gentle dilatation of the smaller vessel edge and suturing with wider bites on the larger vessel. When two graft arteries were encountered, the dominant artery was reconstructed first, and then, the back-bleeding from the second hepatic artery was taken for arterial-blood gas test. Depending on the results, the second artery was anastomosed or ligated (22).

\section{Intraarterial thrombolysis}

All procedures of intraarterial thrombolysis were performed in the angiography suite by intervention radiologists. At first, diagnostic angiography of the celiac trunk was performed. When hepatic artery occlusion was confirmed, intraarterial thrombolysis was performed with a bolus dose of a 100,000-300,000 IU urokinase through a microcatheter. If this was not effective, continuous urokinase infusion at a rate of $30,000 \mathrm{IU} / \mathrm{h}$ was performed for $4-5$ hours.

\section{Immunosuppression}

For all recipients, basiliximab $(20 \mathrm{mg})$ was given intravenously during surgery and on day 4 after LDLT, and maintenance immunosuppressants consisted of corticosteroids, tacrolimus and mycophenolate mofetil. Corticosteroid was administered intravenously during the operation at a dose of $500 \mathrm{mg}$ of methylprednisolone and rapidly tapered over the first week to a dose of $20 \mathrm{mg}$ daily, and was maintained orally for the first 3 to 6 months after operation.

Tacrolimus was started on the next day after surgery with dose adjusted at a trough concentration of $8-12 \mathrm{ng} / \mathrm{mL}$ during the first month and at $5-8 \mathrm{ng} / \mathrm{mL}$ thereafter. Mycophenolate mofetil was administered at a dose of $750 \mathrm{mg}$ twice daily from postoperative day 2 with dosage adjustment according to adverse effects.

For desensitization in the ABO-I LDLT patients, rituximab was given as a single intravenous dose of $\left(300 \mathrm{mg} / \mathrm{m}^{2}\right.$ body surface area) before LDLT. Prior to March 2014, rituximab was administered 2 weeks before surgery, and thereafter 5 to 7 days before LDLT. However, in emergency LDLT, rituximab was given at the very beginning of the operation.

Intravenous immunoglobulin $(0.8 \mathrm{~g} / \mathrm{kg})$ was given on days 1 and 4 after LDLT. Plasmapheresis was conducted before March 2014, and then was discontinued. Neither splenectomy nor graft local infusion was performed (5).

\section{Thromboprophylaxis}

Anticoagulation therapy was maintained during and after LDLT. In donor surgery, heparin (5 IU/kg) was intravenously injected just prior to dividing the hepatic artery. In recipient operation, prostaglandin E1 and antithrombin III were administered for 7-14 days after LDLT. Low-dose aspirin therapy $(100 \mathrm{mg} /$ day $)$ was initiated on the 14th postoperative day and continued for 1 year in patients whose platelet counts were more than $90,000 / \mu \mathrm{L}$.

\section{Infection prophylaxis}

Hepatitis B immunoglobulin and oral antiviral drug were administered in patients with hepatitis B virus. Pegylatedinterferon and ribavirin were given to patients with hepatitis $\mathrm{C}$ virus recurrence after confirmation of hepatitis $\mathrm{C}$ virus RNA levels and elevated liver-enzyme levels. For the prevention of other infections, the patients were also given ticarcillin-clavulanate for one week, fluconazole for one month, and trimethoprim-sulfamethoxazole for one year. Cytomegalovirus prophylaxis was not performed routinely. The cytomegalovirus antigenemia assay was performed twice a week until discharge, weekly until postoperative one month, and once a month thereafter.

\section{Follow-up and surveillance}

Adequate arterial blood flow was evaluated by intraoperative Doppler ultrasonography.

Follow-up Doppler ultrasonography was performed daily during the postoperative two weeks to confirm artery patency. The diagnosis of HAT after LDLT was based on clinical presentation, Doppler ultrasonography findings, dynamic computed tomography, and arteriography.

For follow-up of patients after LDLT, computed tomography scans of the abdomen were checked every 
Table 1 Initial isoagglutinin titers according to combination of ABO blood-type (donor to recipient) in the ABO-I LDLT group

\begin{tabular}{lcc}
\hline ABO type (donor to recipient) & Total $(n=78)$ & Isoagglutinin titers \\
\hline$A \rightarrow B$ & $8(10.3)$ & $64[2-256]$ \\
$A \rightarrow O$ & $18(23.1)$ & $32[4-128]$ \\
$B \rightarrow A$ & $9(11.5)$ & $16[4-32]$ \\
$B \rightarrow O$ & $14(17.9)$ & $32[4-128]$ \\
$A B \rightarrow A$ & $13(16.7)$ & $8[2-32]$ \\
$A B \rightarrow B$ & $14(17.9)$ & $32[2-128]$ \\
$A B \rightarrow O$ & $2(2.6)$ & $16[16-16]$ \\
\hline
\end{tabular}

Data are presented as number (\%) or median [range]. ABO-I, ABO-incompatible; LDLT, living donor liver transplantation.

3 months during the first 2 years, every 4 months during the third year, and biannually thereafter.

\section{Statistical analysis}

Data are supplied as number (\%) or median (interquartile range) unless otherwise specified. Chi-square test or Fisher's exact test for categorical variables was used for comparisons between the two groups. Student's $t$-test or Mann-Whitney $\mathrm{U}$ test was used for continuous variables according to the normality of the distribution.

$\mathrm{P}$ values $<0.05$ were considered statistically significant. Calculations were made using the SPSS 24.0 statistical software package (IBM, Inc., Chicago, IL, USA) and R 3.3.3 (https://www.r-project.org).

\section{Results}

A total of 378 patients underwent LDLT during the study period. Of those, ABO-I LDLT was performed in 78 consecutive patients without suitable $\mathrm{ABO}-\mathrm{C}$ living donors rituximab was administered a median of 7 days ( $0-14$ days) before surgery. Plasmapheresis was done a median of 2 times (1-3 times) before LDLT in the initial 21 ABO-I LDLT patients. The data on the blood type combinations with each titer of donor and recipient were summarized in Table 1 . A to $\mathrm{O}$ in 18 patients $(23.1 \%)$ was the most common combination of the ABO type of donor to recipient. The initial isoagglutinin titer prior to desensitization had the median value of 32 (range, 2-256). The titer decreased to 8 (range, $0-16$ ) at the time of surgery.

The other 300 patients with ABO-C LDLT constituted the comparator group. The baseline demographic and perioperative data of donors and recipients are presented in Table 2. Details of the hepatic artery reconstruction are described in Table 3. There were no significant differences for all the variables.

After a median follow-up period of 40.2 months (range, 12.3-84.5 months), HAT was developed in 11 (2.9\%) patients, 2 and 9 patients in ABO-I and ABO-C LDLT groups, respectively, which showed no significant difference between the two groups $(\mathrm{P}=0.84)$. All were categorized into early HAT (Table 4). Actually, every HAT was diagnosed within 24 hours after LDLT. Especially, 5 (1 ABO-I, 4 ABO-C) patients had intraoperative unresolved HAT despite multiple attempts of redo anastomosis. Immediate revascularization by intraarterial thrombolysis was successfully employed in 8 patients. Surgical revision of anastomosis and retransplantation were performed in 1 and 2 patients, respectively, following failed endovascular treatment. No one-year mortality was related to HAT in the two groups. In these patients with HAT, 7 (63.6\%) patients developed biliary complications; 5 (1 ABO-I, 4 ABO-C) biliary stricture, 1 (1 ABO-C) fistula, and 1 (1 ABO-C) mixed stricture and fistula. All those patients were managed with percutaneous transhepatic biliary drainage.

Of those with HAT, the overall survival rate was $63.6 \%$ (7/11) with a median survival of 51.2 months (range, 7.8-66.9 months). One ABO-C patient had nonanastomotic biliary stricture and died of hepatic failure 37 months after LDLT. Two deaths (1 ABO-C, 1 ABO-I) were caused by tumor recurrence 7.8 and 38.3 months after LDLT, respectively. One ABO-C patient committed suicide because of depression 66.9 months after LDLT.

The overall outcomes including biliary complications made no significant differences between the two groups. No antibody-mediated rejection was observed in the two study groups. Acute cellular rejection occurred in one ABO-C 
Table 2 Baseline demographic and perioperative characteristics

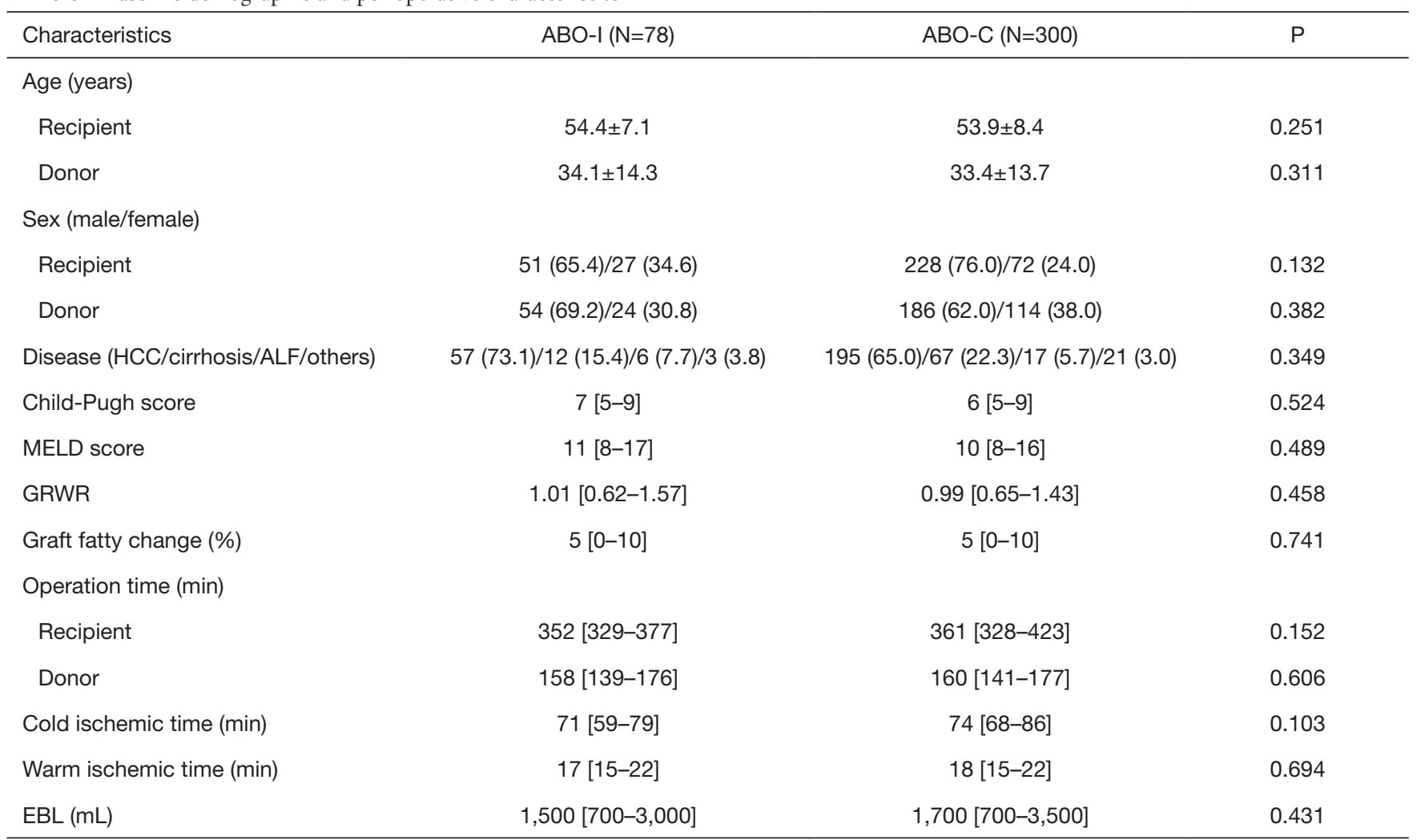

Data are presented as number (\%), mean \pm standard deviation or median [interquartile range]. ABO-I, ABO-incompatible; ABO-C, ABOcompatible; ALF, acute liver failure; HCC, hepatocellular carcinoma; EBL, estimated blood loss; GRWR, graft-to-recipient body weight ratio; MELD, model for end-stage liver disease.

LDLT patient. The mortality within 30 postoperative days occurred in 3 ABO-C LDLT patients, each from asphyxia by foreign body, septic shock, and heart failure. The 1-, 3-, and 5 -year overall survival rates in the ABO-I and ABO-C LDLT groups were $91.1 \%, 83.5 \%, 77.5 \%$ and $94.2 \%$, $86.3 \%, 80.5 \%$, respectively $(\mathrm{P}=0.47)$.

\section{Discussion}

To the best of our knowledge, this is the first comparative analysis between ABO-I and ABO-C LDLTs with a special reference to HAT. ABO-incompatibility showed no statistical significance as a risk factor for HAT. Study results also indicated that rituximab was not associated with increased incidence of HAT in ABO-I LDLT, and that this desensitizing regimen was effective in preventing antibody-mediated rejection. In this analysis, regardless of ABO-compatibility, HAT happened in LDLT patients with the overall incidence of $2.9 \%$. And, ABO-I LDLT showed comparable outcomes to ABO-C LDLT from the standpoint of HAT outcomes and patient survival rates, consequently abrogating $\mathrm{ABO}$-incompatibility as one of nonsurgical risk factors for the development of HAT.

The observation of similar outcomes in HAT among patients according to $\mathrm{ABO}$-incompatibility deserves attention. The overall agreement is that hyperacute rejection can occur in patients of ABO-I LDLT though the liver is known to be more resistant to hyperacute rejection than the kidney or heart. The $\mathrm{ABO}$ Blood group antigens are not only expressed on the surface of blood cells, but also on the surface of the endothelium of vessels (23). Vascular endothelium of hepatic allografts may continue to express donor blood group antigens up to 150 days after transplantation (24). Therefore, the graft of ABO-I LDLT may be more susceptible to HAT (25).

Although plasmapheresis was used for the initial 20 patients in this study, rituximab was administered in all ABO-I LDLT patients without exception. Therefore, the single major difference in terms of immunosuppression was the use of rituximab between the two groups. Rituximab is a 
Table 3 Characteristics of hepatic arteries involved in anastomoses

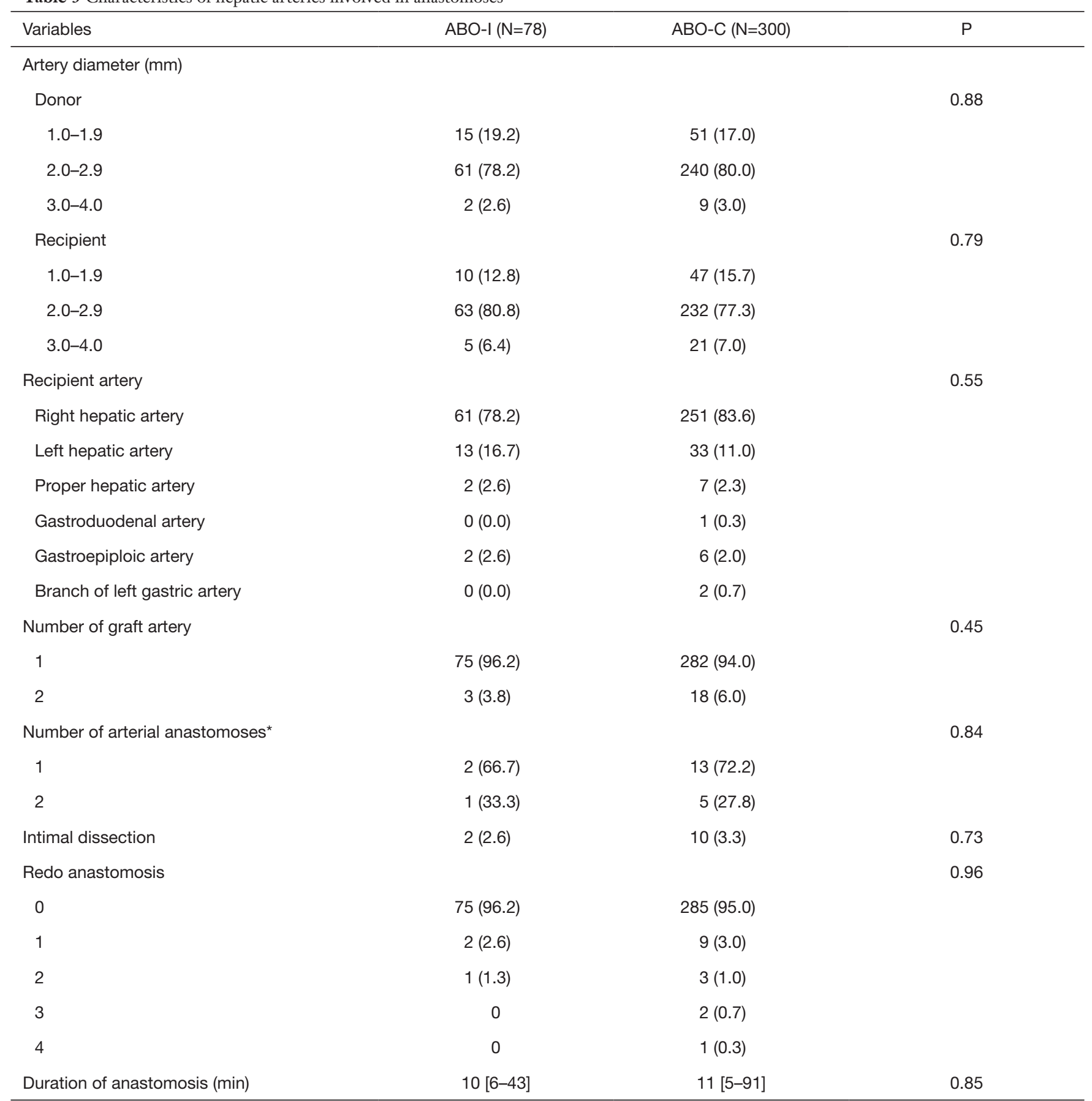

Data are presented as number (\%) or median [range]. *, in cases with two graft hepatic arteries. ABO-I, ABO-incompatible; ABO-C, ABOcompatible.

monoclonal chimeric human anti-CD20 antibody that can deplete peripheral blood B cells (26). So, from the similar outcomes between the two groups, it can be concluded that depletion of B cells by rituximab doesn't affect HAT incidence after operation.

Risk factors for HAT have been investigated in several studies. Hypercoagulable state such as factor V Leiden mutation, cytomegalovirus mismatch, prolonged operative 
Table 4 HAT and overall outcomes in ABO-I vs. ABO-C LDLTs

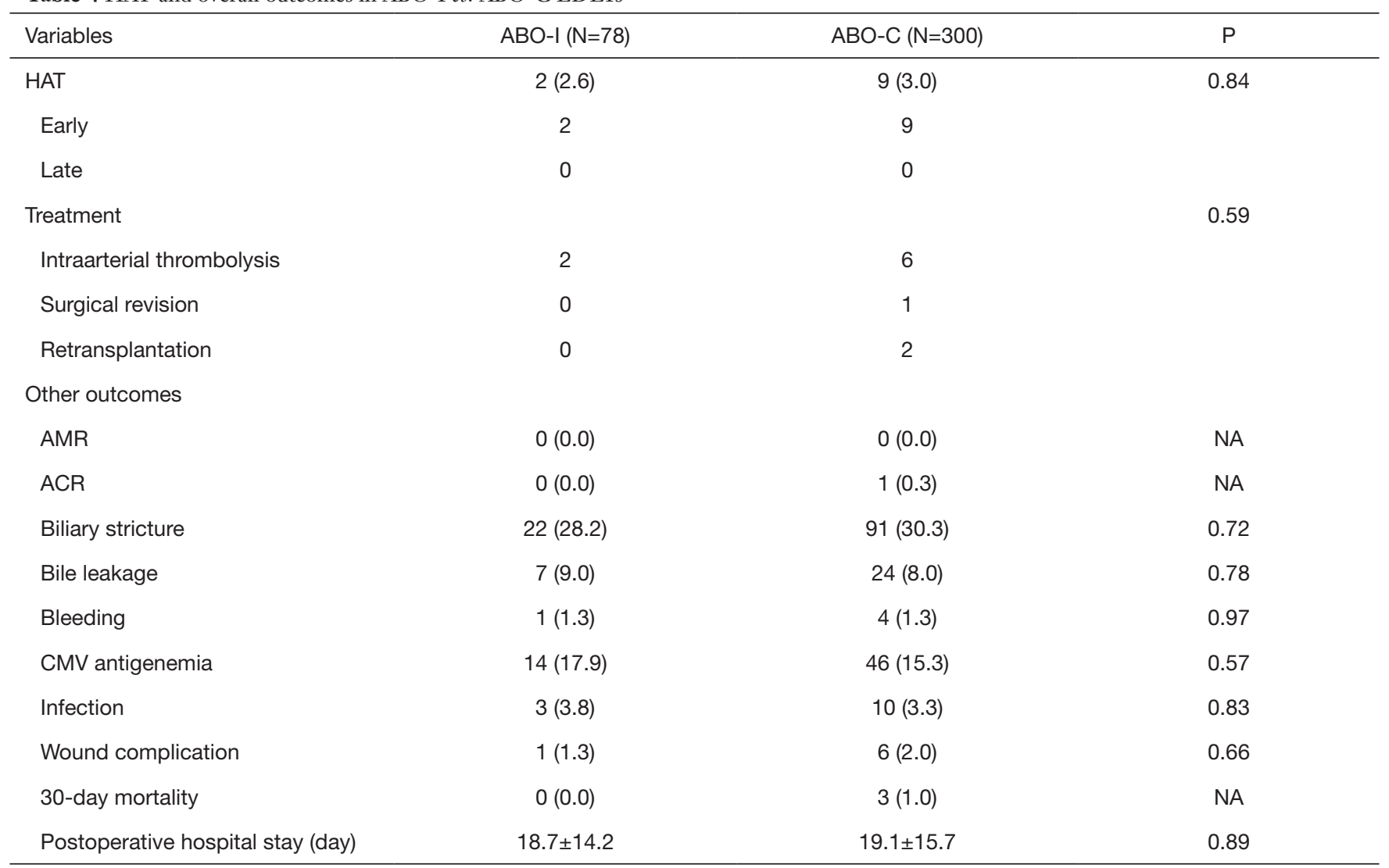

Data are presented as number (\%) or mean \pm standard deviation. LDLT, living donor liver transplantation; ABO-I, ABO-incompatible; ABO-C, ABO-compatible; AMR, antibody-mediated rejection; ACR, acute cellular rejection; HAT, hepatic artery thrombosis; CMV, cytomegalovirus; N/A, not applicable.

and ischemic times, episode of acute rejection, arterial diameter discrepancy between donor and recipient, intraoperative transfusion, pretransplant transarterial chemoembolization, donor age, and a long donor graft artery were associated with HAT occurrence (1,2,27-32). Several techniques have also been reported for avoiding HAT with a focus only on technical factors, but not on non-operative factors such as ABO-incompatibility. The mechanism of HAT development is still elusive and is believed to be multifactorial including both surgical and nonsurgical factors.

All the hepatic artery anastomoses in this study were performed by a single surgeon who had specially dedicated to microscopic vascular surgery and had already previous experience with more than 300 hepatic artery anastomoses in LDLT in addition to hepatic artery dissection for anastomosis in donor and recipient surgery as a main operator before this study. And, in all recipients, hepatic artery anastomosis was done with the same technique. So, all the surgical factors including the learning curve effect can be ruled out as possible causes of HAT. Given no significant differences in the baseline demographic and perioperative characteristics that can represent nonsurgical factors, therefore, the only notable difference between the two groups was $\mathrm{ABO}$-incompatibility. So the study design wouldn't lose validity and objectivity in pursuit of the study purpose.

In this study, all ABO-I LDLTs were the only option for curative treatment because patients had no compatible donor. In our center, less than $5 \%$ of all transplant patients undergo deceased donor liver transplantation, and most patients undergo LDLT, mirroring the present circumstances of a single institution in a region that has a low rate of deceased donor organ recovery. Our ABO-I LDLT program was started in 2012, adding rituximab, immunoglobulin, and plasmapheresis to the 
conventional immunosuppression used in ABO-C LDLT. And plasmapheresis was discontinued since March 2014. This streamlined protocol could stave off the high costs and complications by other desensitizing procedures, such as local graft infusion or splenectomy. This rituximabbased regimen showed parallel outcomes between ABO-I and ABO-C LDLTs (5). The satisfactory outcome was the linchpin on which ABO-I LDLT could be continued as one curative treatment option for patients awaiting liver transplant.

This study has a major limitation of its retrospective, non-randomized design from a single institution. The general consensus is that the gold standard for evaluating the effect of different interventions on outcomes is randomized controlled trials. However, because of the difficulty of patient recruitment and current preference for ABO-C over ABO-I LDLT in clinical practice, it is almost impossible to perform randomized controlled trial comparing ABO-I and ABO-C LDLT. However, this study recruited a relatively homogeneous group of adult LDLT patients, which revealed all covariates giving no difference of the baseline patient characteristics and operative details, and accordingly eliminated any confounding effects of other perioperative variables except for $\mathrm{ABO}$ incompatibility even without utilizing propensity score matching.

In conclusion, this study showed that the overall incidence of HAT in LDLT was low with current improved surgical technique, and that revealed no detrimental impact of ABO incompatibility on the overall outcomes including HAT by demonstrating comparable results between ABO-I and ABO-C LDLT patients, taking the stand that ABO-I LDLT can be performed in patients with no compatible donor awaiting liver transplant.

\section{Acknowledgments}

None.

\section{Footnote}

Conflicts of Interest: The authors have no conflicts of interest to declare.

Ethical Statement: The authors are accountable for all aspects of the work in ensuring that questions related to the accuracy or integrity of any part of the work are appropriately investigated and resolved. The Institutional Review Board of National Cancer Center, Korea approved this study, and informed consent was waived because this was a retrospective study (IRB number: NCC2019-0087).

\section{References}

1. Bekker J, Ploem S, de Jong KP. Early hepatic artery thrombosis after liver transplantation: a systematic review of the incidence, outcome and risk factors. Am J Transplant 2009;9:746-57.

2. Pastacaldi S, Teixeira R, Montalto P, et al. Hepatic artery thrombosis after orthotopic liver transplantation: a review of nonsurgical causes. Liver Transpl 2001;7:75-81.

3. Egawa H, Teramukai S, Haga H, et al. Impact of rituximab desensitization on blood-type-incompatible adult living donor liver transplantation: a Japanese multicenter study. Am J Transplant 2014;14:102-14.

4. Song GW, Lee SG, Hwang S, et al. Section 15. A desensitizing protocol without local graft infusion therapy and splenectomy is a safe and effective method in ABOincompatible adult LDLT. Transplantation 2014;97 Suppl 8:S59-66.

5. Kim SH, Lee EC, Shim JR, et al. A simplified protocol using rituximab and immunoglobulin for $\mathrm{ABO}$ incompatible low-titre living donor liver transplantation. Liver Int 2018;38:932-9.

6. Dada R, Zekri J, Ramal B, et al. Acute jugular vein thrombosis during rituximab administration: Review of the literature. J Oncol Pharm Pract 2016;22:165-9.

7. Arunprasath P, Gobu P, Dubashi B, et al. Rituximab induced myocardial infarction: A fatal drug reaction. J Cancer Res Ther 2011;7:346-8.

8. Sultan Y, Bussel A, Maisonneuve P, et al. Potential danger of thrombosis after plasma exchange in the treatment of patients with immune disease. Transfusion 1979;19:588-93.

9. Meneu-Diaz JC, Moreno-Gonzalez E, Garcia Garcia I, et al. Hepatic allograft arterialization by means of the gastroduodenal bifurcation (branch patch) as a prognostic factor. Transplantation 2004;77:1513-7.

10. Kim SH, Cho SY, Park SJ, et al. Learning curve for livingdonor liver transplantation in a fledgling cancer center. Transpl Int 2009;22:1164-71.

11. Kim SH, Kim YK, Lee SD, et al. Selection and outcomes of living donors with a remnant volume less than $30 \%$ after right hepatectomy. Liver Transpl 2013;19:872-8.

12. Hoon Kim S, Duke Lee S, Kyu-Kim Y, et al. Living donors ages 60 or older in right lobe living donor liver transplantation. Transplantation 2015;99:e16-7. 
13. Kim SH, Lee SD, Kim YK, et al. Impact of IntraAbdominal Adhesion on Living Donor Right Hepatectomy. Am J Transplant 2016;16:1788-94.

14. Bhattacharjya S, Gunson BK, Mirza DF, et al. Delayed hepatic artery thrombosis in adult orthotopic liver transplantation-a 12-year experience. Transplantation 2001;71:1592-6.

15. Stange BJ, Glanemann M, Nuessler NC, et al. Hepatic artery thrombosis after adult liver transplantation. Liver Transpl 2003;9:612-20.

16. Kim SH, Kim YK. Improving outcomes of living-donor right hepatectomy. Br J Surg 2013;100:528-34.

17. Kim SH, Kim YK, Lee SD, et al. The Impact of a Surgical Protocol for Enhanced Recovery on Living Donor Right Hepatectomy: A Single-Center Cohort Study. Medicine (Baltimore) 2016;95:e3227.

18. Kim SH, Lee SD, Kim YK, et al. Pushing the frontiers of living donor right hepatectomy. World J Gastroenterol 2014;20:18061-9.

19. Kim SH, Lee EC, Lee SD, et al. Ligation and cut as a method for bile duct division in living donor right hepatectomy. Liver Transpl 2017;23:448-56.

20. Kim SH, Cho SY, Lee KW, et al. Upper midline incision for living donor right hepatectomy. Liver Transpl 2009;15:193-8.

21. Kim SH, Kim YK. Living donor right hepatectomy using the hanging maneuver by Glisson's approach under the upper midline incision. World J Surg 2012;36:401-6.

22. Kim SH, Na BG, Lee EC, et al. Arterial blood gas test to decide whether to reconstruct single or both the arteries in living donor liver transplantation. Hepatobiliary Surg Nutr 2018;7:440-2.

23. Rydberg L. ABO-incompatibility in solid organ

Cite this article as: Kim SH, Park J, Park SJ. Impact of ABOincompatibility on hepatic artery thrombosis in living donor liver transplantation. Ann Transl Med 2019;7(22):625. doi: 10.21037/atm.2019.11.34 transplantation. Transfus Med 2001;11:325-42.

24. Sanchez-Urdazpal L, Batts KP, Gores GJ, et al. Increased bile duct complications in liver transplantation across the ABO barrier. Ann Surg 1993;218:152-8.

25. Wu J, Ye S, Xu X, et al. Recipient outcomes after ABOincompatible liver transplantation: a systematic review and meta-analysis. PLoS One 2011;6:e16521.

26. Genberg H, Hansson A, Wernerson A, et al. Pharmacodynamics of rituximab in kidney allotransplantation. American journal of transplantation 2006;6:2418-28.

27. Tanyel FC, Ocal T, Balkanci F, et al. The factor V Leiden mutation: a possible contributor to the hepatic artery thrombosis encountered after liver transplantation in a child. J Pediatr Surg 2000;35:607-9.

28. Abdelaziz O, Hosny K, Amin A, et al. Endovascular management of early hepatic artery thrombosis after living donor liver transplantation. Transpl Int 2012;25:847-56.

29. Panaro F, Ramos J, Gallix B, et al. Hepatic artery complications following liver transplantation. Does preoperative chemoembolization impact the postoperative course? Clin Transplant 2014;28:598-605.

30. Wu L, Zhang J, Guo Z, et al. Hepatic artery thrombosis after orthotopic liver transplant: a review of the same institute 5 years later. Exp Clin Transplant 2011;9:191-6.

31. Herrero A, Souche R, Joly E, et al. Early Hepatic Artery Thrombosis After Liver Transplantation: What is the Impact of the Arterial Reconstruction Type? World J Surg 2017;41:2101-10.

32. Oh CK, Pelletier SJ, Sawyer RG, et al. Uni- and multi-variate analysis of risk factors for early and late hepatic artery thrombosis after liver transplantation. Transplantation 2001;71:767-72. 